\title{
Descrição dos imaturos e primeiro registro de planta hospedeira de Charidotis gemellata Boheman (Coleoptera, Chrysomelidae, Cassidinae) ${ }^{1}$
}

\author{
Flávia Rodrigues Fernandes ${ }^{2,3} \&$ Zundir José Buzzi²,4
}

${ }^{1}$ Contribuição ${ }^{\circ} 1656$ do Departamento de Zoologia, Universidade Federal do Paraná.

${ }^{2}$ Laboratório de Taxonomia e Biologia de Chrysomelidae, Departamento de Zoologia, Universidade Federal do Paraná, Caixa Postal 19020, 81531-980 Curitiba-PR, Brasil.

${ }^{3}$ Programa de Pós-graduação em Entomologia (UFPR). flarfer@gmail.com

${ }^{4}$ zbuzzi@ufpr.br

\begin{abstract}
Description of immatures and first record of host plant for Charidotis gemellata Boheman (Coleoptera, Chrysomelidae, Cassidinae). The egg, fifth instar larva and pupa of Charidotis gemellata are described and illustrated. The examined material was collected in Centro Politécnico Campus of the Universidade Federal do Paraná in Curitiba/ PR, Brazil, where adults of Charidotis gemellata were found on the leaves of Pithecoctenium crucigerum Gentry L. (Bignoniaceae). This is the first record of a host plant for C. gemellata and also the first record for the Paraná state (Brazil).
\end{abstract}

KEYWORDS. Cassidini; larva; morphology; Pithecoctenium crucigerum; pupa.

RESUMO. Descrição dos imaturos e primeiro registro de planta hospedeira de Charidotis gemellata Boheman (Coleoptera, Chrysomelidae, Cassidinae). O ovo, a larva de quinto instar e pupa de Charidotis gemellata são descritos e ilustrados. O material examinado foi coletado no Campus do Centro Politécnico (UFPR), Curitiba/PR, Brasil; onde foram encontrados adultos de Charidotis gemellata Boheman sobre folhas de Pithecoctenium crucigerum Gentry L. (Bignoniaceae). Este é o primeiro registro de planta hospedeira para $C$. gemellata e também o primeiro registro para o estado do Paraná.

PALAVRAS-CHAVE. Cassidini; larva; morfologia; Pithecoctenium crucigerum; pupa.

O gênero Charidotis foi erigido por Boheman (1855) para alocar 45 espécies. Spaeth (1936) incluiu 150 espécies, distribuídas pela América Central e do Sul. No catálogo de Blackwelder (1946) estão relacionadas 170 espécies. O catálogo mais atual de Cassidinae lista 161 espécies para o gênero (Borowiec, 1999). Charidotis miniata Boheman, 1855 foi designada por Hincks (1952) como a espécie tipo.

Até o momento foram descritos os imaturos de 06 espécies de Charidotis. Fiebrig (1910) descreveu os imaturos de $C$. auroguttata Boheman, 1855; C. clypeolata Boheman, 1855; C. gibbipennis Spaeth, 1910; C. mansueta (Boheman, 1855); C. punctatostriata Boheman, 1856; C. ocularis Boheman, 1855 e o ciclo de vida de $C$. pygmaea (Klug, 1829) foi estudado por Day et al. (1999).

Fiebrig (1910) relata as plantas hospedeiras das espécies a seguir: C. clypeolata Boheman, 1855 em Tecoma ipe Mart. (Bignoniaceae); C. gibbipennis Spaeth, 1914 em Anisomeris obtusa (Cham. \& Schlechtd.) K. Sch. (Rubiaceae); C. mansueta Boheman, 1855 em Pyrostegia venusta Miers (Bignoniaceae); C. ocularis Boheman, 1855 em Arrabidaea triplinervia $\mathrm{H}$. Baill. (Bignoniaceae) e em Patagonula americana L. (Boraginaceae) e C. punctatostriata Boheman, $1855 \mathrm{em}$ Pyrostegia venusta Miers (Bignoniaceae). C. auroguttata Boheman, 1855 ocorre em Bignonia exoleta Vell (Bignoniaceae) de acordo com Lima (1955) e em Macfadyena ungüis-cati (L.) Gentry (Bignoniaceae) de acordo com Fiebrig (1910) e Williams
(2002). De acordo com Flowers \& Janzen (1997) C. costaricea Spaeth, 1936 vive em Guettarda macrosperma Donn. Sm. (Rubiaceae). Silva et al. (1968) relatam a ocorrência de $C$. flavicans Boheman, 1855 em Pyrostegia venusta Miers (Bignoniaceae) e de C. marginella (Fabricius, 1775) em Eucalyptus saligna Smith (Myrtaceae). Windsor et al. (1992) relatam C. abrupta Boheman, 1855 em Anemopaegma orbiculatum (Jacq.) A. DC. (Bignoniaceae); C. leprieuri (Boheman, 1855) em Phryganocydia corymbosa Bureau (Bignoniaceae); C. nevermanni Spaeth, 1936 em Stizophyllum riparium (Kunth) Sandwith (Bignoniaceae) e C. pustulata Champion, 1894 em Liabum bourgeaui Hieron (Asteraceae). De acordo com Day et al. (1999) C. pygmaea (Klug, 1829) pode ser encontrada em Lantana camara $L$. (Verbenaceae), $L$. montevidensis (Spreng.) Briq. (Verbenaceae), L. tiliaefolia Cham. (Verbenaceae) e L. fucata Lindl. (Verbenaceae). Buzzi (2002) relata a planta hospedeira de C. terenosensis Buzzi, 2002 como Memora peregrina (Miers) Sandwith (Bignoniaceae). De acordo com Buzzi (1994), C. tesselata Boheman, 1855 alimenta-se de Macfadyena ungüis-cati (L.) Gentry (Bignoniaceae).

O estudo das fases ontogenéticas do ciclo evolutivo de insetos se reveste de grande importância pois permite, muitas vezes, o estabelecimento de sinonímias e é de grande importância na identificação de espécies crípticas (Buzzi, 1996).

Visando a possível utilização de espécies de Charidotis 
como agentes de controle biológico de plantas invasoras, verifica-se um crescente interesse pelo ciclo de vida desse grupo como visto em Day et al. (1999), Day \& McAndrew (2002) e Williams (2002).

Charidotis gemellata foi descrita por Boheman (1855), e distribui-se nos estados de Santa Catarina e Rio Grande do Sul (Borowiec, 2002).

Visando um maior conhecimento do ciclo evolutivo de $C$. gemellata, este trabalho, tem como objetivo descrever os imaturos e relatar uma espécie de planta hospedeira de $C$. gemellata.

\section{MATERIALE MÉTODOS}

Adultos de C. gemellata foram coletados sobre folhas de Pithecoctenium crucigerum Gentry L. (Bignoniaceae), identificada no herbário do Departamento de Botânica da Universidade Federal do Paraná (UPCB).

Os adultos foram acondicionados em gaiola confeccionada com garrafa plástica (tipo PET), seccionada ao meio, cuja abertura foi vedada com tecido fino (tipo tule) e presa com elástico.

$\mathrm{Na}$ gaiola foram acondicionados ramos da planta hospedeira P. crucigerum Gentry L. dentro de um cilindro plástico, de $5 \mathrm{~cm}$ de altura e $3 \mathrm{~cm}$ de diâmetro, com algodão umedecido. Realizou-se a limpeza e a troca do alimento a cada dois dias. Os ovos retirados foram acondicionados em placa de Petri sobre um disco de papel filtro umedecido com água. Após a eclosão, as larvas foram transferidas para outra placa de Petri e alimentadas com folhas de P. crucigerum Gentry L. até atingirem o estágio de pupa.

Todo o experimento foi realizado em sala climatizada com temperatura de $25^{\circ} \pm 1^{\circ} \mathrm{C}$, umidade relativa $70 \pm 10 \%$, e com fotofase de 12 horas.

As larvas de quinto ínstar e pupas foram fixadas em KahleDietrich para as descrições e elaboração dos desenhos. A terminologia utilizada para as descrições das estruturas morfológicas do ovo seguiu Fiebrig (1910). A terminologia utilizada para as descrições das estruturas morfológicas das larvas e pupa baseou-se em Buzzi (1996). As mensurações e ilustrações foram efetuadas com o auxílio de um microscópio estereoscópio WILD M5, equipado com câmara clara e ocular micrométrica de 120 divisões e as medidas transformadas em milímetros.

\section{RESULTADOS}

Ovo

(Fig. 1)

Tegumento liso, aproximadamente 2,1 vezes mais longo que seu maior diâmetro, de coloração amarelo-ocre. Recobrindo o ovo há uma cobertura presa na parte posterior, assemelhando-se a um cilindro cortado ao meio longitudinalmente; de parede muito fina, com ondulações transversais, de cor âmbar e no centro com uma faixa longitudinal vermelho-acastanhada. Os bordos da cobertura

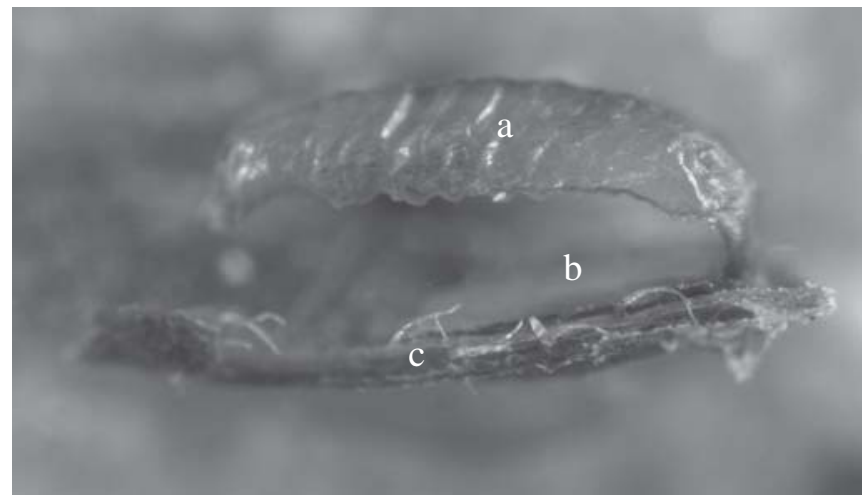

Fig. 1. Charidotis gemellata. Ovo, vista lateral. a, cobertura; b, ovo; c, substrato.

são dobrados para baixo, sendo que esta ultrapassa o comprimento do ovo.

Comprimento e largura médios do ovo, em mm, respectivamente: 1,34 (Amplitude: 1,14 - 1,62; s: 0,119); 0,64 (Amplitude: 0,56 - 0,72; s: 0,054). Comprimento e largura médios, em mm, da cobertura do ovo, respectivamente: 1,67 (Amplitude: 1,28 - 1,90; s: 0,119); 0,72 (Amplitude: 0,62 - 0,88; s: 0,073).

\section{Larva de quinto ínstar}

(Figs. 2 e 3)

Corpo cerca de 2,2 vezes mais longo que sua maior largura; levemente achatado dorso-ventralmente; lados subparalelos, convergindo levemente para frente a partir do metatórax e fortemente para trás a partir do sexto tergo. Com 15 pares de escolos, subiguais em comprimento, assim dispostos: dois pares na margem anterior do protórax, três pares aos lados do mesotórax, dois pares aos lados do metatórax e oito pares aos lados do abdômen, um par em cada segmento, sendo o oitavo voltado para trás. Primeiro par de escolos bifurcado, sendo o ramo anterior com comprimento médio de $1,20 \mathrm{~mm}$ e o ramo posterior com $1,24 \mathrm{~mm}$. O restante dos escolos possuem as seguintes medidas de comprimento médio, em mm: 1,08; 1,08 ; 0,$84 ; 1,00 ; 0,76 ; 0,88 ; 0,96 ; 0,92 ; 0,80 ; 0,76 ; 0,76 ; 1,20 ; 1,36$; 1,24. Os escolos possuem cerdas laterais grossas, fortes e eretas; com no máximo sete e no mínimo três a cada lado. Furca anal do oitavo segmento abdominal alcança o primeiro segmento abdominal, quando voltada para frente.

Tegumento amarelo-pardo, exceto os escolos castanhoclaros e o pronoto com um par de manchas castanho escuras, laterais, cobrindo aproximadamente $2 / 3$ de cada metade da placa pronotal sendo a porção antero-lateral mais escura. Pronoto com tegumento glabro. No lado ventral, poucas cerdas no meio dos esternos torácicos e um tufo de cerdas no meio dos três primeiros esternos abdominais.

Cabeça subarrendondada, em vista frontal (Fig. 3, a); em vista lateral, ligeiramente achatada. Com microesculturações triangulares ântero-vertidas no tegumento. Parietais cerca de 1,5 vezes mais longos que largos; com forte depressão central. Quetotaxia simétrica, sendo de cada lado: uma cerda frontal, 

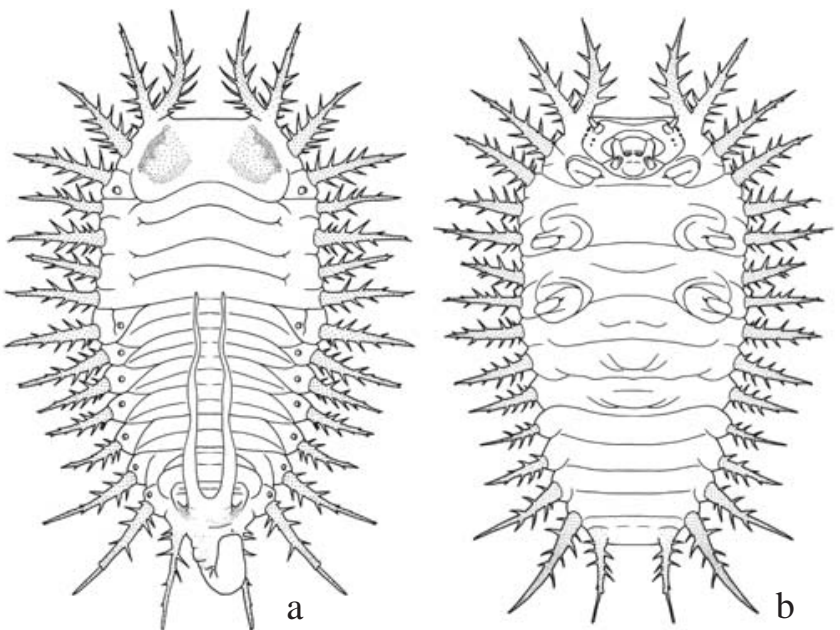

Fig. 2. Charidotis gemellata. Larva de $5^{\circ}$ ínstar. a, vista dorsal e b, vista ventral.

próxima à sutura interparietal; dois pares de cerdas frontais, eqüidistantes, dispostas em linha transversal no nível dos ocelos; três pares de cerdas frontais, eqüidistantes, dispostas em linha transversal no nível logo acima da margem anterior do clípeo. Largura média da cápsula cefálica: $0,95 \mathrm{~mm}$ (amplitude: 0,88 - 1,04 mm; s: 0,044).

Antenas com artículo basal anelar e o distal subcônico, cerca de três vezes mais longo que o basal, com processos sensoriais apicais. Ocelos em número de seis, sobre pequenos tubérculos, dispostos aos pares, logo atrás das antenas. Clípeo com superfície lisa; cerca de 2,5 vezes mais largo que seu comprimento mediano. Labro (Fig. 3, b) aproximadamente duas vezes mais largo que seu comprimento mediano; margem posterior levemente convexa, margem anterior bissinuada, com forte chanfro mediano e sete cerdas, a cada lado, em linha transversal, logo atrás da margem; a cada metade uma cerda na linha mediana próxima à margem posterior e uma cerda disposta em linha transversal próxima à margem lateral. Mandíbulas (Fig. 3, d) com forte côndilo na base e quatro dentes no ápice, sendo o dente basal com dois dentículos; duas cerdas externas (Fig. 3, a), uma cerda basal próxima à margem externa e uma cerda mediana menor próxima à margem interna. Maxilas (Fig. 3, c) com cardo alongado com duas cerdas na margem externa; estipe menor que os palpos; mala globosa com três cerdas na margem anterior; palpo com dois artículos, subiguais em comprimento, o apical subcônico e o basal anelar com três cerdas em linha. Lábio (Fig. 3, c) com área do mento e submento membranosa, a cada lado com três cerdas dispostas em triângulo próximo aos palpos; palpos uniarticulados e subcônicos, ápice com processos sensoriais.

Placa pronotal ampla, abrangendo quase toda a porção dorsal do pronoto, dividida ao meio por suave sulco; margem anterior levemente arqueada e posterior sinuosa na porção mediana.

Mesonoto e metanoto semelhantes na forma, em torno de 3,4 vezes mais largos que longos; divididos por sulco transversal em pré-tergito e pós-tergito; mesonoto, lateroanteriormente, com espiráculo e três pares de escolos.

Tergos torácicos e abdominais divididos em pré-tergitos e pós-tergitos por um sulco mediano transversal que, no tórax, não alcança as margens laterais e no abdômen, não alcança a linha dos espiráculos. Tergos I-VIII abdominais com um par de espiráculos próximos à base dos escolos que diminuem gradativamente de diâmetro, espiráculo do oitavo tergo muito atrofiado.

Fêmures e tíbias (Fig. 3, e) de comprimentos iguais, apresentando microesculturações triangulares ântero-vertidas; tarsúngulo com dente laminar e cerda basal; fêmur glabro; tíbia com uma cerda basal dorsal, três cerdas medianas ventrais e duas cerdas apicais ventrais; tarso com uma cerda apical dorsal, uma cerda mediana dorsal e duas cerdas apicais ventrais.

Esternos abdominais muito alargados e curtos; o segundo
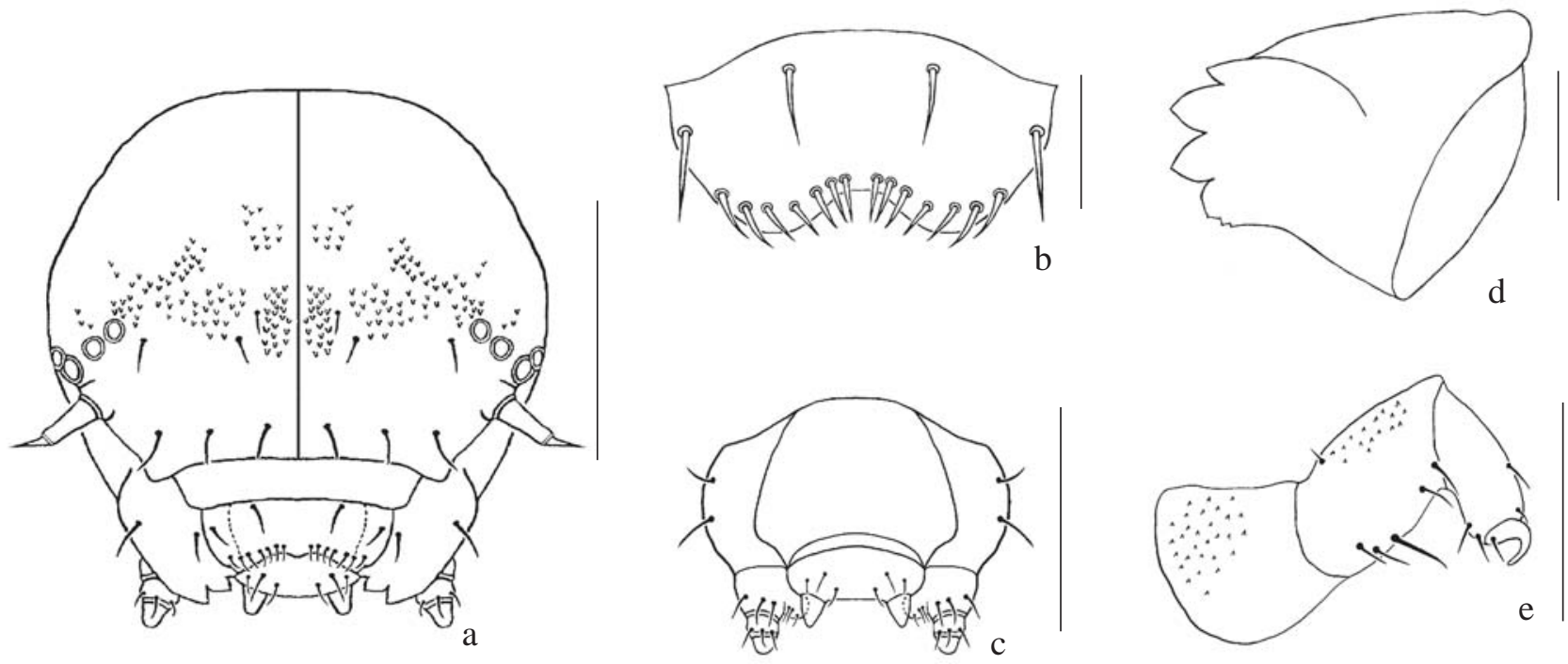

Fig. 3. Charidotis gemellata. Larva de $5^{\circ}$ ínstar. a, cápsula cefálica (escala $=5 \mathrm{~mm}$ ); b, labro (escala $=1 \mathrm{~mm}$ ); d, mandíbula, vista interna (escala $=1 \mathrm{~mm}) ; \mathrm{c}$, maxila + lábio $($ escala $=5 \mathrm{~mm})$; e, perna média $($ escala $=5 \mathrm{~mm})$. 
cerca de 4,0 vezes mais largo que longo; os demais semelhantes, porém, a partir do quarto, gradualmente estreitados para trás; o nono e o décimo segmentos formam o tubo anal.

\section{Pupa \\ (Fig. 4)}

Corpo dorsalmente convexo, ventralmente aplanado; cerca de 1,6 vezes mais longo que sua maior largura, excluídos os escolos. Tegumento amarelo, glabro. Abdômen com lados subparalelos; do primeiro ao quinto tergo com as margens convergindo anteriormente e a partir do sexto tergo convergindo posteriormente. Comprimento médio da pupa: 4,39 $\mathrm{mm}(4,80-3,76 \mathrm{~mm} ; \mathrm{s}: 0,2547)$.

Pronoto cerca de duas vezes mais largo do que longo; margem anterior semicircular, com 11 pares de projeções espiniformes; segundo e terceiro pares com cerdas curtas na metade basal; porção central do pronoto mais elevada que as bordas, rebaixadas lateralmente; profundo sulco mediano longitudinal, nos $2 / 3$ posteriores do pronoto, não atingindo a margem posterior e um sulco curto e raso cortando-o perpendicularmente, formando uma cruz na porção mediana posterior do pronoto; margem posterior com curta e ampla projeção mediana.

Mesonoto com escutelo saliente e projetado posteriormente com duas depressões lateralmente dispostas; mesoescuto com margem posterior com projeção mediana aguda. Élitros voltados para trás, alcançando a margem anterior do quinto esterno abdominal. Metanoto com porção central pouco mais longa que a do mesonoto; margem anterior com ampla sinuosidade mediana.

Abdômen com primeiro tergo cerca de 8,1 vezes mais largo que longo, excluídos os escolos; tergos dois a cinco semelhantes ao primeiro; sexto tergo ao oitavo, gradualmente estreitados para trás; oitavo tergo cerca de 4,1 vezes mais largo que longo. Aos lados com oito pares de escolos abdominais, um par por segmento, diminuindo gradativamente de tamanho (com as seguintes mensurações, em mm: 1,72; $1,48 ; 1,36 ; 1,16 ; 0,76 ; 0,72 ; 0,60 ; 0,28)$; do primeiro ao quinto aplanados, subtriangulares e projetados ântero-lateralmente; os demais, subcilíndricos, paralelos à margem do corpo e projetados posteriormente, sendo o oitavo escolo observado somente em vista ventral. Os cinco primeiros escolos com cerdas laterais grossas, fortes e eretas e com, no máximo, cinco e no mínimo três cerdas laterais. Espiráculos posicionados látero-dorsalmente, próximo à base dos escolos, sendo um par por segmento, do primeiro ao quinto tergo abdominal. Entre a linha dos espiráculos e a linha mediana dorsal, uma fileira de pequenas depressões, uma em cada tergo, que diminuem de tamanho gradativamente, do primeiro ao sexto tergo.

Cabeça em vista ventral, cerca de 1,4 vezes mais longa que larga; ao longo do meio com sulco raso; no ápice com dois lobos curtos e achatados. Antenas divergentes e alcançam a articulação fêmuro-tibial da perna média; segmentação evidente. Área clípeo-labral, cerca de 1,2 vezes mais larga que seu comprimento mediano. Mandíbulas, em concha, cerca de 1,6 vezes mais longas que largas; face externa fortemente
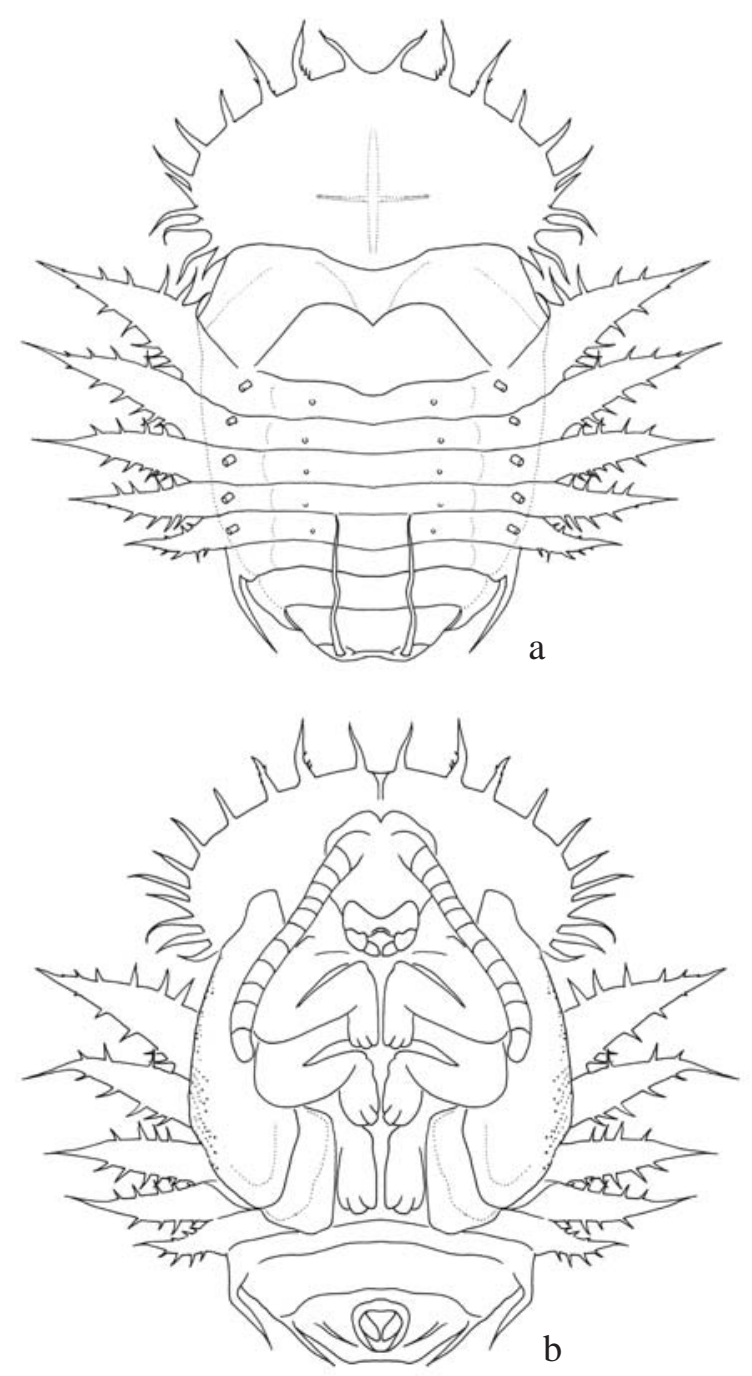

Fig. 4. Charidotis gemellata. Pupa. a, vista dorsal; b, vista ventral.

convexa. Palpos digitiformes; os maxilares pouco mais longos e grossos que os labiais.

Perna anterior com fêmur e tíbia de mesmo comprimento e achatados de encontro ao corpo; tarsos com segmentação pouco evidente. Perna média e posterior semelhantes a anterior; a posterior com fêmur e tíbia encobertos pelos élitros, exceto o tarso.

Esternos abdominais, de um a quatro, totalmente encobertos pelos élitros; do quinto ao oitavo visíveis; oitavo com comprimento uniforme, quase tão longo aos lados como no meio, abertura anal transversal. Furca anal com ramos medindo cerca de $1,40 \mathrm{~mm}$, voltados anteriormente, alcançando o quarto tergo; na base afastados um do outro cerca de $0,30 \mathrm{~mm}$.

Material examinado. Dez espécimes adultos de Charidotis gemellata Boheman, 1855 foram depositados na Coleção de Entomologia Pe. Jesus Santiago Moure, Departamento de Zoologia, Universidade Federal do Paraná (DZUP). 


\section{DISCUSSÃO}

No presente trabalho foi identificada e confirmada como planta hospedeira Pithecoctenium crucigerum (L.) A. Gentry, (Bignoniaceae), popularmente conhecida como pente-demacaco, de distribuição do México até Argentina e Uruguai (Lorenzo-Cáceres, 2002). Esta espécie é o primeiro registro de planta hospedeira para Charidotis gemellata.

Os ovos são fixados horizontalmente no substrato por um dos lados de maior comprimento, sobre ambas as faces das folhas e sobre pecíolos da planta hospedeira, postos isoladamente ou em grupo de no máximo 20. Tal disposição é semelhante ao encontrado em Charidotis auroguttata (Williams, 2002) e difere do encontrado em C. punctatostriata que põe seus ovos sempre em grupos e apenas nos pecíolos de sua planta hospedeira (Buzzi, 1988).

Comparando a morfologia do ovo de Charidotis gemellata com a de C. auroguttata apresentada por Williams (2002), as duas espécies apresentam uma extensa cobertura membranosa presa na parte posterior do ovo que cobre toda a extensão dorsal exposta.

As larvas de C. gemellata alimentam-se tanto de folhas jovens como velhas da planta hospedeira e possuem cinco estádios. A independência da idade da folha na alimentação e o número de estádios larvais também é observado para $C$. auroguttata (Williams, 2002) e C. pygmaea (Day \& McAndrew, 2002).

$\mathrm{O}$ anexo exúvio-fecal da larva permanece na pupa, o que foi também observado em $C$. gibbipenis e $C$. punctatostriata (Buzzi, 1988). No mesmo trabalho o autor afirma que este fato ocorre provavelmente com todas as espécies de Charidotis.

A larva de quinto ínstar de $C$. gemellata permanece alguns dias sem se alimentar antes de empupar e a pupa prende-se nas folhas da planta hospedeira. C. auroguttata também possui um período sem alimentação pré-pupal, porém, diferentemente de $C$. gemellata, esta espécie empupa nos pecíolos da planta hospedeira e no solo (Williams, 2002).

Agradecimentos: Ao CNPq pela concessão das bolsas de estudo. À MSc. Elaine Della Giustina Soares pelas sugestões nas versões anteriores do manuscrito. À graduanda de Engenharia Florestal Luana Rodrigues pela confecção dos desenhos das figuras 2 e 4 .

\section{REFERÊNCIAS}

Blackwelder, R. E. 1946. Checklist of the coleopterous insects of Mexico, Central America, The West Indies and South America. Bulletin of Smithsonian Institute USNM 185: 551-763.
Boheman, C. 1855. Monographia Cassididarum. Tomus tertius, Holmiae, 543 p.

Borowiec, L. 1999. A world catalogue of the Cassidinae (Coleoptera: Chrysomelidae). Biologica Silesiae. 476 p.

Borowiec, L. 2002. New records of Neotropical Cassidinae, with description of three new species (Coleoptera: Chrysomelidae). Genus 13: 43-138.

Buzzi, Z. J. 1988. Biology of Neotropical Cassidinae, p. 559-580. In: P. Jolivet; E. Petitpierre \& T. H. Hsiao (eds.), Biology of Chrysomelidae. London, Kluwer Academic Publischers. 615 p.

Buzzi, Z. J. 1994. Host plants of Neotropical Cassidinae. p. 205-212. In: P. H. Jolivet; M. L. Cox \& E. Petitpierre (eds.), Novel aspects of the biology of Chrysomelidae. Kluwer Academic Publichers.

Buzzi, Z. J. 1996. Morfologia dos imaturos e ciclo evolutivo de Anacassis dubia (Boheman), A. fuscata (Klug), A. languida (Boheman), A. phaeopoda Buzzi e A. punctulata (Klug) (Coleoptera, Chrysomelidae, Cassidinae). Revista Brasileira de Zoologia 13: 215-289.

Buzzi, Z. J. 2002. Uma nova espécie de Charidotis Boheman de Terenos, Mato Grosso do Sul, Brasil (Coleoptera, Chrysomelidae, Cassidinae). Revista Brasileira de Zoologia 19: 335-337.

Day, M. D.; B. W. Willson \& H. F. Nahrung. 1999. The life history and host range of the golden lantana beetle, Charidotis pygmaea (Coleoptera: Chrysomelidae), a biological control agent for Lantana montevidensis (Verbenaceae). Biocontrol Science and Technology 9: 347-354.

Day, M. D. \& T. D. McAndrew. 2002. Status of Charidotis pygmaea (Coleoptera: Chrysomelidae) as a Biological Control Agent of Lantana montevidensis (Verbenaceae) in Australia. Biological Control 23: 27-34.

Fiebrig, K. 1910. Cassiden und Cryptocephaliden Paraguays. Zoologische Jahbucher 12: 161-264.

Flowers R. W. \& H. Janzen. 1997. Feeding records of Costa Rican leaf beetles (Coleoptera: Chrysomelidae). Florida Entomologist 80: 334-366.

Hincks, W. D. 1952. The genera of the Cassidinae (Coleoptera: Chrysomelidae). Transactions of the Royal Entomological Society of London 103: 327-358.

Lorenzo-Cáceres, J. M. S. 2002. Bignonias trepadoras cultivadas en España. Parjap 25: 36-41.

Silva, A. G. d'A.; C. R. Gonçalves; D. M. Galvao; A. J. Gonçalves; J. Gomes; M do N. Silva \& L. de Simoni. 1968. Quarto catálogo dos insetos que vivem nas plantas do Brasil. Seus parasitos e predadores. Ministério da Agricultura, Laboratório Central de Patologia Vegetal. Rio de Janeiro. Parte II, 1o Tomo, 622 p.

Spaeth, F. 1936. Bestimmungstabelle der Gattung Charidotis. Sbornik Entomologického Oddìlení Národního Musea v Praze 14 66-99.

Williams, H. E. 2002. Life history and laboratory host range of Charidotis auroguttata (Boheman) (Coleoptera: Chrysomelidae), the first natural enemy released against Macfadyena Unguis-Cati (L.) Gentry (Bignoniaceae) in South Africa. The Coleopterists Bulletin 56: 299-307.

Windsor, D. M.; E. G. Riley \& H. P. Stockwell. 1992. An introduction to the biology and systematics of Panamanian Tortoise Beetles (Coleoptera: Chrysomelidae: Cassidinae). In: D. Quintero \& A. Aiello (eds.). Insects of Panama and Mesoamerica, Selected studies. Oxford Univ. Press, Oxford, New York, Tokyo. 372$391 \mathrm{p}$.

Recebido em 03/11/2006; aceito em 20/03/2007 